\title{
The Treatment of Facial Asymmetry with Botulinum Toxin: Current Concepts, Guidelines, and Future Trends
}

\author{
Izolda Heydenrych ${ }^{1}$ \\ ${ }^{1}$ Department of Dermatology, Cape Town Cosmetic Dermatology \\ Centre, Central Park on Esplanade, Century City, Cape Town, \\ South Africa
}

Indian J Plast Surg:2020;53:219-229

\begin{abstract}
Address for correspondence Izolda Heydenrych, MBChB, MMed (Derm), BSc Hons (Nucl Med), Department of Dermatology, Cape town Cosmetic Dermatology Centre, Central Park on Esplanade, Postnet Suite 136, Century City, Cape Town 7442, South Africa (e-mail: izoldaheydenrych@gmail.com).
\end{abstract}

\begin{abstract}
Keywords

- Bell's palsy

- facial asymmetry

- facial nerve paralysis

- onabotulinum toxin $\mathrm{A}$

- quality of life

This article will describe facial asymmetry secondary to facial nerve paralysis (FNP), and review current concepts, guidelines, and future trends. Despite the increasing use of botulinum toxin (BoNTA) in treating FNP, ideal dosage, timing, and additional therapies are not unequivocally established. Facial asymmetry significantly impacts quality of life (QOL) by strongly affecting self-perception and social interactions; injectables may mediate great clinical improvement. This article provides practical guidelines for the use of BoNTA and provides schemes for accurate assessment and documentation. A systematic, stepwise approach is recommended with methodical assessment, meticulous placement, conservative dosage, and careful follow-up. Future trends include the potential use of newly developed toxins, muscle modification with fillers, improved imaging techniques, and targeted QOL studies. Hopefully, a growing number of aesthetic injectors may become technically proficient and join multidisciplinary teams for managing FNP.
\end{abstract}

\section{Introduction}

Vocation is said to be the place where our deep gladness meets the world's deep need (Frederick Buechner, Wishful Thinking, 1993). As such, the treatment of facial nerve palsy (FNP) offers a true vocational niche in the field of aesthetic medicine.

Facial asymmetry comprises a vast clinical spectrum. This article will focus on patients exhibiting asymmetry and muscle dysfunction secondary to FNP. ${ }^{1}$ Despite a recent systematic review validating botulinum toxin type A (BoNTA) injections as a helpful, minimally invasive technique for restoring facial symmetry at rest and in animation, a surprisingly small minority of aesthetic injectors treat this condition. Hesitance may well be ascribed to the uncertainty of approach in a highly complex patient cohort. Although the optimum dose, treatment intervals, and optimal adjunctive therapies are not unequivocally established, this article will discuss current concepts, guidelines, and future trends. ${ }^{2}$ The hope is that interest might be kindled for the treatment of a deserving group of patients, and that injectables may increasingly be utilized by a growing cohort of proficient physicians; also, in geographical areas without existing multidisciplinary centers.

\section{Current Concepts}

The multivariate etiology of FNP includes tumor resections (-Fig. 1), base of skull injuries, Ramsey Hunt syndrome, and Bell's palsy. Clinical presentation varies according to the location of the facial nerve lesion ( - Fig. 2).

Characteristic symptoms ensue because the facial nerve carries not only motor fibers, but also supplies autonomic innervation to the lacrimal and submandibular glands, taste to the anterior two-thirds of the tongue, and sensation to parts of the ear. ${ }^{3}$ While immune mechanisms, infection and
License terms

()(1) $\odot \circledast$ 


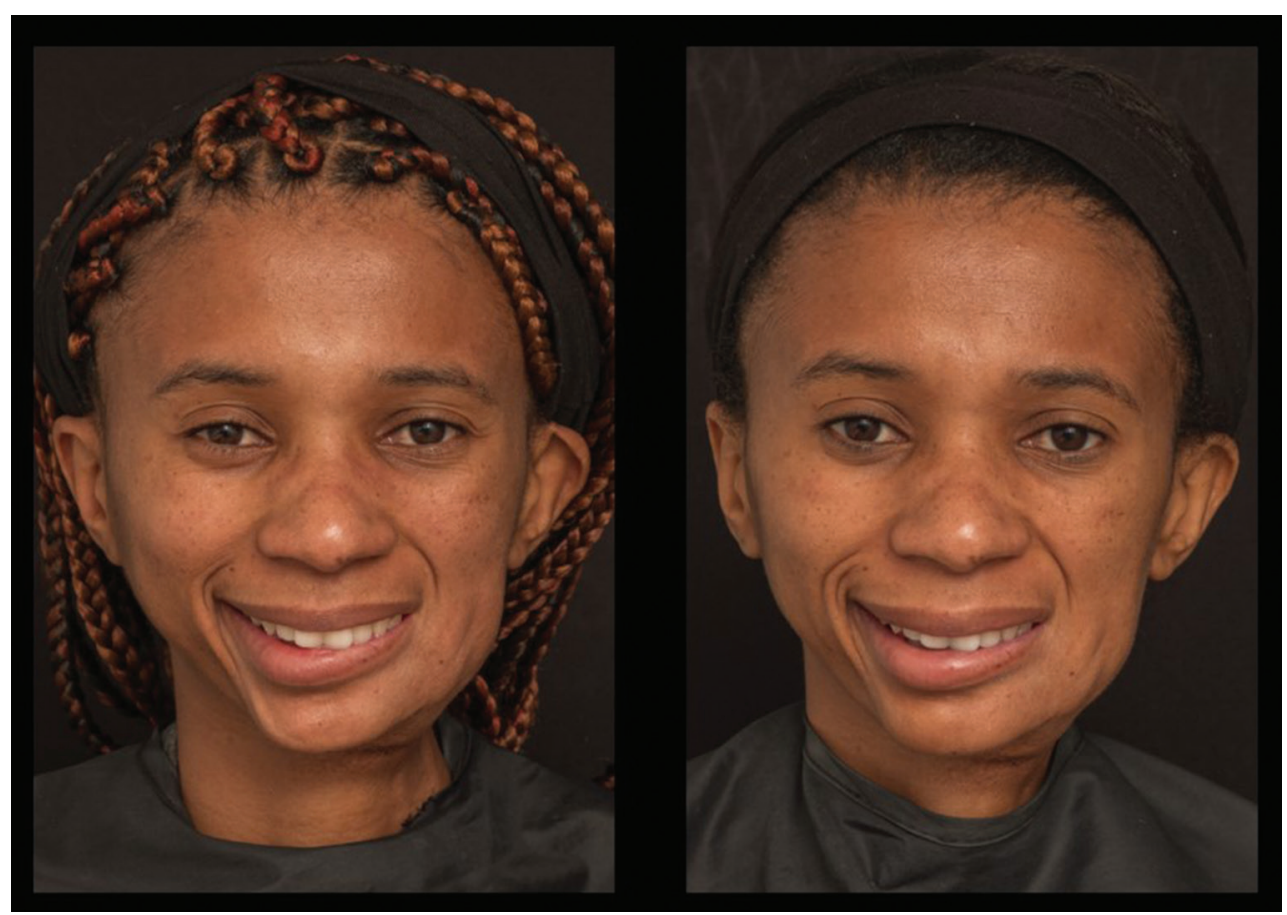

Fig. 1 Patient with longstanding facial nerve paralysis (FNP) after resection of an osteogenic sarcoma, before and two weeks after first treatment with botulinum toxin (BoNTA). Note the improvement in mentalis synkinesis, more symmetrical smile, and improved eye apertures on animation.

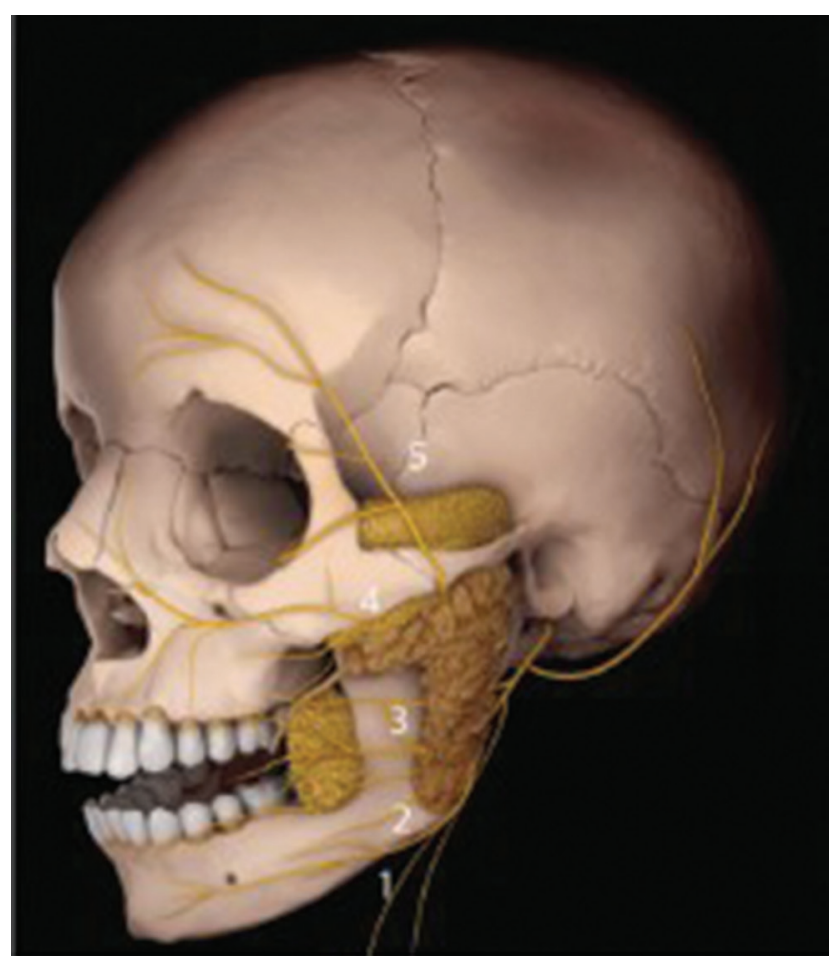

Fig. 2 Branches of the facial nerve VII, all of which may be affected by facial nerve paralysis (FNP). ${ }^{5}$

ischemia are potential contributors to the development of Bell's palsy, the exact cause remains unclear and may be further elucidated by improved understanding of intraaxonal signaling molecules and the molecular mechanisms underlying Wallerian degeneration. ${ }^{4}$ Recent guidelines advocate steroid monotherapy for acute Bell's palsy, with controversy regarding the benefit of combined antivirals and corticosteroids. $^{5}$

Current treatments for nonflaccid FNP include nonsurgical interventions such as BoNTA, surgery, and biofeedback therapies like neuromuscular retraining (NMR) techniques. Where flaccid facial paralysis persists, BoNTA injections into the nonparalyzed side may improve symmetry. ${ }^{6}$

\section{Multispecialty Approach}

For patients with long-lasting, incomplete recovery, a combination of functional (swallowing, speech, eye closure, nasal patency), aesthetic, and psychological factors should be considered. Interdisciplinary cooperation, amongst a wide range of subspecialties, is proving to be most effective, with a combination of NMR, targeted BoNTA, and selective surgical intervention mitigating long-standing impairment.

\section{Quality of Life in FNP}

In addition to objective examination of facial movement, subjective evaluation of quality of life (QOL) is required. Two-thirds of the 1 in 60 patients affected by FNP during their lifetime will demonstrate incomplete recovery, with initial flaccid FNP eventually complicated by contralateral hyperkinesis or ipsilateral synkinesis. ${ }^{2}$ The chronic sequelae of incomplete or aberrant facial nerve regeneration have far-reaching implications for QOL. ${ }^{2}$

Various factors contribute to QOL outcomes, and careful clinical observation assists in optimal therapeutic decisions. ${ }^{7}$ 
The degree of FNP is the key predictor for QOL. Other factors include the following:

- Congenital or malignant etiologies associated with higher QOL.

- Viral etiology, anxiety, obesity, chronic pain, and radiation therapy associated with lower QOL

- Diabetes mellitus is a critical factor for facial rehabilitation after acute FNP, as uncontrolled glucose levels may impede neural regeneration. ${ }^{8}$

All-inclusive care for both psychosocial and functional sequelae is vital. ${ }^{3}$ Aesthetic concerns strongly influence both self-perception and social interaction, with functional concerns including decreased peripheral vision. In addition to the physical effects of paralysis, patients demonstrate lower social functioning, low self-confidence, and phobic avoidance of social interactions. Anxiety and depression are more common in long-term FNP, and early psychological counseling is vital to decrease morbidity in those at risk of poorer outcomes. $^{3}$

Interestingly, a recent cross-sectional study found patients with left-sided FNP to have far lower QOL scores than rightsided FNP. Possible explanations include the superiority of the right hemisphere both in recognizing facial emotions and in processing emotional information. The left visual field, which is projected on the cortex of the right hemisphere, may thus be important in emotional impression. ${ }^{3}$

\section{BoNTA in Acute FNP}

A recent study utilizing BoNTA in the treatment of acute FNP showed potential efficacy as an alternative in patients with contraindications to existing medical or surgical treatments. ${ }^{8}$ Despite current knowledge of ipsilateral muscle facilitation and improved symmetrical function through decreasing contralateral hyperkinesis, the use of BoNTA in acute FNP has not previously been extensively studied. A recent study demonstrated a single dose of BoNTA on the nonparalyzed side of 18 patients with acute facial paralysis, to cause marked improvement in facial asymmetry 1 month after injection. ${ }^{8}$ Study patients presented with total neural degeneration and House-Brackmann (HB) grading IV-VI along with underlying etiologies, including Ramsey Hunt syndrome (zoster oticus). Strengthened ipsilateral muscle function and improved $\mathrm{HB}$ and Sunnybrook (SB) grading were documented 6 months postinjection. ${ }^{8}$ Because treatment of acute FNP should be conducted during the regenerative and not degenerative period, BoNTA was timed from 1 to 6 months after initial onset, as this is considered a sufficient period for cessation of Wallerian degeneration and onset of neural regeneration. ${ }^{9}$

Critical assessment of medical history and individual variability in muscle strength and anatomy is vital. Severe pain may predict herpes zoster with development of a vesicular eruption, progression to Ramsay Hunt syndrome, and poorer prognosis for recovery. ${ }^{3}$ Supportive care during the acute phase of FNP is of cardinal importance, and as physicians we have the privilege of offering encouragement during this vulnerable stage (-Fig. $\mathbf{3}$ ).

\section{The Assessment of FNP}

Facial paralysis is an intricate condition which may be exceedingly challenging to describe, measure, and follow-up. A recent comprehensive review of clinician-rated scales revealed no

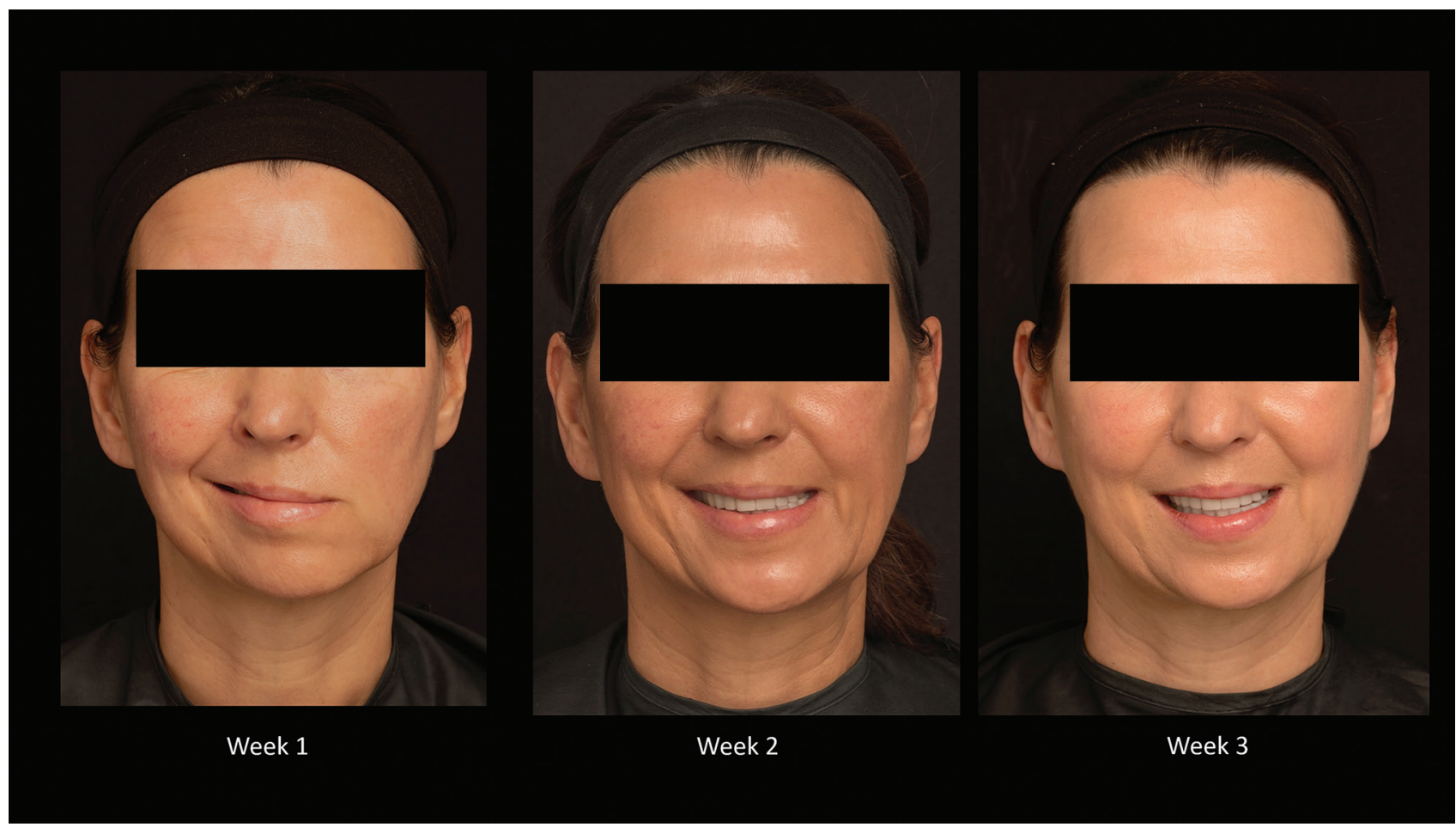

Fig. 3 Record of weekly recovery in a patient with Ramsey Hunt syndrome, demonstrating excellent recovery. 
single ideal method; the emerging trend is for extensive assessment and documentation through clinician-graded scales, QOL tools, patient-reported outcomes, and measures of function and disfigurement. Graphs, pictures, and scoring systems are invaluable, while regular use of systematic ratings adds finesse to clinical observation ( $\mathbf{- T a b l e s} \mathbf{1}$ and $\mathbf{2}$ ).

\section{Ocular Sequelae after FNP}

Optimal eyecare is of paramount importance after FNP, with ophthalmological consultation highly recommended. Eyelid asymmetry is frequent in severe facial weakness as the eyelid maintenance and recalibration system may be overwhelmed. ${ }^{12}$ There may be either ptosis or retraction on the FNP side, depending on factors such as ipsilateral synkinesis and contralateral ptosis. Other potential ocular sequelae include the following ${ }^{13}$ :

\section{- Poor or incomplete blink.}

Table 1 Scoring system for eye closure ${ }^{10}$

\begin{tabular}{|l|l|l|}
\hline Grade & Designation & Description \\
\hline 1 & Poor & $\begin{array}{l}\text { No eye closure; maximum scleral } \\
\text { show }\end{array}$ \\
\hline 2 & Fair & $\begin{array}{l}\text { Poor eye closure; two-thirds scleral } \\
\text { show }\end{array}$ \\
\hline 3 & Moderate & $\begin{array}{l}\text { Partial eye closure; one-third scleral } \\
\text { show }\end{array}$ \\
\hline 4 & Good & $\begin{array}{l}\text { Almost full eye closure; minimum } \\
\text { scleral show }\end{array}$ \\
\hline 5 & Excellent & Full eye closure; no scleral show \\
\hline
\end{tabular}

Table 2 Aesthetic and functional grading system used for smile ${ }^{11}$

\begin{tabular}{|l|l|l|}
\hline Grade & Result & Description \\
\hline 1 & Poor & Deformity; no contraction \\
\hline 2 & Fair & No symmetry; minimum contraction \\
\hline 3 & Moderate & $\begin{array}{l}\text { Moderate symmetry and contraction; mass } \\
\text { movement }\end{array}$ \\
\hline 4 & Good & Symmetry; nearly complete contraction \\
\hline 5 & Excellent & $\begin{array}{l}\text { Symmetrical smile; teeth visible; complete } \\
\text { contraction }\end{array}$ \\
\hline
\end{tabular}

- Brow ptosis.

- Lagophthalmos.

- Upper eyelid retraction: possibly related to pre-existing contralateral ptosis, greater Hering forces on the FNP side, unopposed levator palpebrae superioris (LPS) action, or stiff actin-myosin cross-bridges in the levator superioris muscle.

- Eyelid ptosis: Heterogeneous presentation, not exclusively associated with synkinesis, usually present later in the disease. Ipsilateral ptosis with underlying synkinesis may benefit from targeted BoNTA therapy.

Diminished tear production is of specific concern as it may lead to ${ }^{12}$ :

- Compromised ocular surface protection.

- Exposure keratitis, ulceration or corneal abrasion.

- Ultimate visual impairment.

Ophthalmological collaboration is vital in prescribing optimal lubricants.

\section{History, Clinical Assessment, and Documentation}

Insightful facial assessment, with elucidation of the main dynamic and anatomical concerns, is vital. Standardized photography and videography are important for baseline documentation and monitoring treatment efficacy.

\section{Grading Systems}

Despite a lack of consensus regarding appropriate scales for clinician-graded and patient-reported scales, the HB scale and SB system (facial nerve grading scale) are the most frequently used. ${ }^{14,15}$ Physicians should select a preferential system and document consistently (- Table 3, - Figs. 4 and $\mathbf{5}$ ).

\section{Photography}

Clinical photography and videography of standardized facial movements constitute mandatory pre- and posttreatment assessment. ${ }^{4}$ The nine standard photographic views include face at rest, brow raise, gentle and tight eye closure, nasal creasing/snarl, open and closed-lip smile, puckering, whistle, and lower lip depression. ${ }^{4}$ Importantly, common synkinesis

Table 3 The House-Brackmann scale for severity of peripheral FNP ${ }^{14}$

\begin{tabular}{|l|l|l|l|l|l|}
\hline Grade & Description & At rest & $\begin{array}{l}\text { Forehead } \\
\text { innervation }\end{array}$ & Lid closure & Mouth innervation \\
\hline I & Normal & Normal & Normal & Normal & Normal \\
\hline II & Mild dysfunction & Normal & Reduced & Nearly normal & Nearly normal \\
\hline III & Moderate dysfunction & Normal & Still barely possible & Still barely possible & Reduced \\
\hline IV & $\begin{array}{l}\text { Moderately severe } \\
\text { dysfunction }\end{array}$ & Normal & None & Incomplete & Asymmetrical \\
\hline V & Severe dysfunction & Asymmetrical & None & Incomplete & Asymmetrical \\
\hline VI & Total paralysis & Complete loss of tone & None & None & None \\
\hline
\end{tabular}

Abbreviation: FNP, facial nerve palsy. 


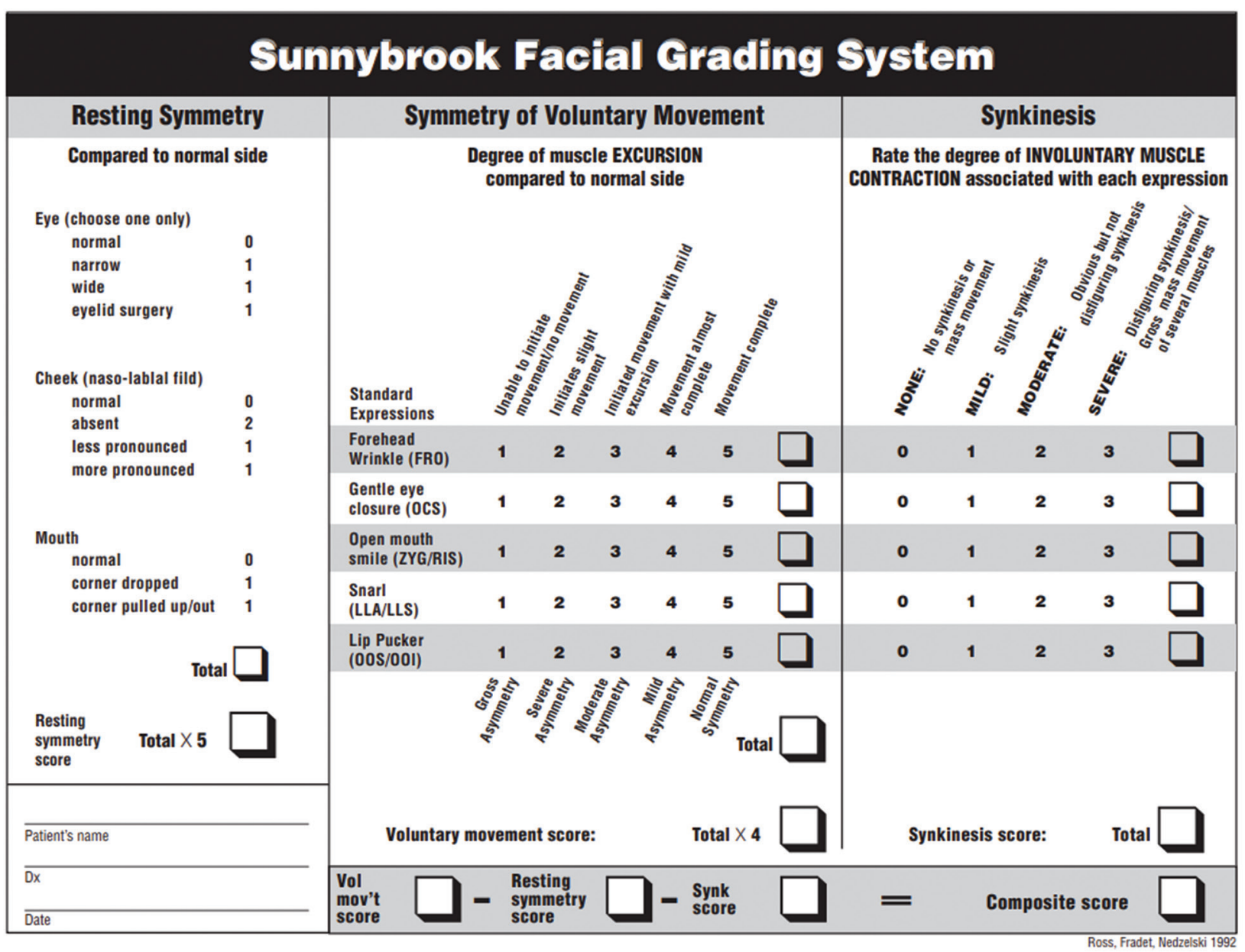

Fig. 4 Example of the Sunnybrook Facial Grading System sheet. ${ }^{15}$

triggers such as forceful eye closing or lip-puckering should be identified.

\section{Synkinesis}

The involuntary, aberrant cocontraction of muscles is due to ipsilateral abnormal facial nerve regeneration, has an incidence of 15 to $55 \%$ after FNP, and generally presents at/after 6 months. ${ }^{2,6}$ Assessment and treatment are challenging, with synkinesis representing a major predictor of QOL. Persistence leads to impaired facial expressions, abnormal resting tone, and impaired self-image. Profoundly affected social interactions may cause unpredictable psychological consequences. ${ }^{17}$ Synkinesis can also induce peripheral visual impairment. ${ }^{2}$ Barely noticeable synkinesis often causes significant distress. Restoration of coordinated, symmetrical muscle contractions, with reduced frequency of abnormal volleys, improves normal muscle function and enables natural facial expressions. ${ }^{18}$ BoNTA as a treatment modality has unequivocally proven to reduce morbidity and significantly improve QOL. ${ }^{6}$

\section{Mechanisms and Patterns of Synkinesis}

Abnormal peripheral facial nerve regeneration and heightened excitability within the facial nucleus are potential causes. ${ }^{6}$ Postparalytic synkinesis may involve agonists groups, such as orbicularis oculi (OO) contraction on Duchenne smiling, while simultaneous contraction of antagonist muscles, such as zygomaticus major and depressor anguli oris or platysma, may cause apparent paralysis. ${ }^{2}$ Multiple degrees and patterns of ocular, oral, and cervical synkinesis, dyskinesis, and hyperkinesis are described, ranging from subtle to completely disfiguring..$^{19}$ Both oral-ocular and ocular-oral synkinesis lead to deepening of the nasolabial fold, further accentuating facial asymmetry. Synkinesis is named by combining the intended motor group, followed by the unintended motor group.

Common synkinetic patterns include the following ${ }^{20}$ :

- Oral-ocular:

- Intended smile or grimace causes involuntarily eyelid closure or spasm.

- Lip puckering evokes synkinesis of eye.

- Smiling causes hypertonicity of the nasolabial fold. 


\section{Massachusetts Eye and Ear eFace}
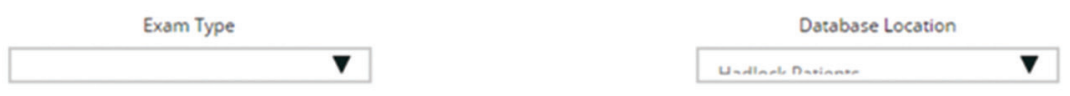

\section{$\underline{\text { Static Parameters }}$}

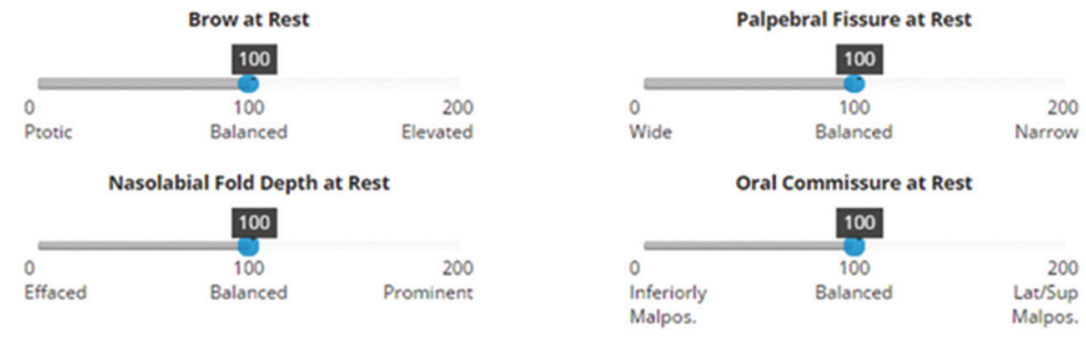

If Nasolabial Fold Orientation At Rest is Not Discernable, Check Box
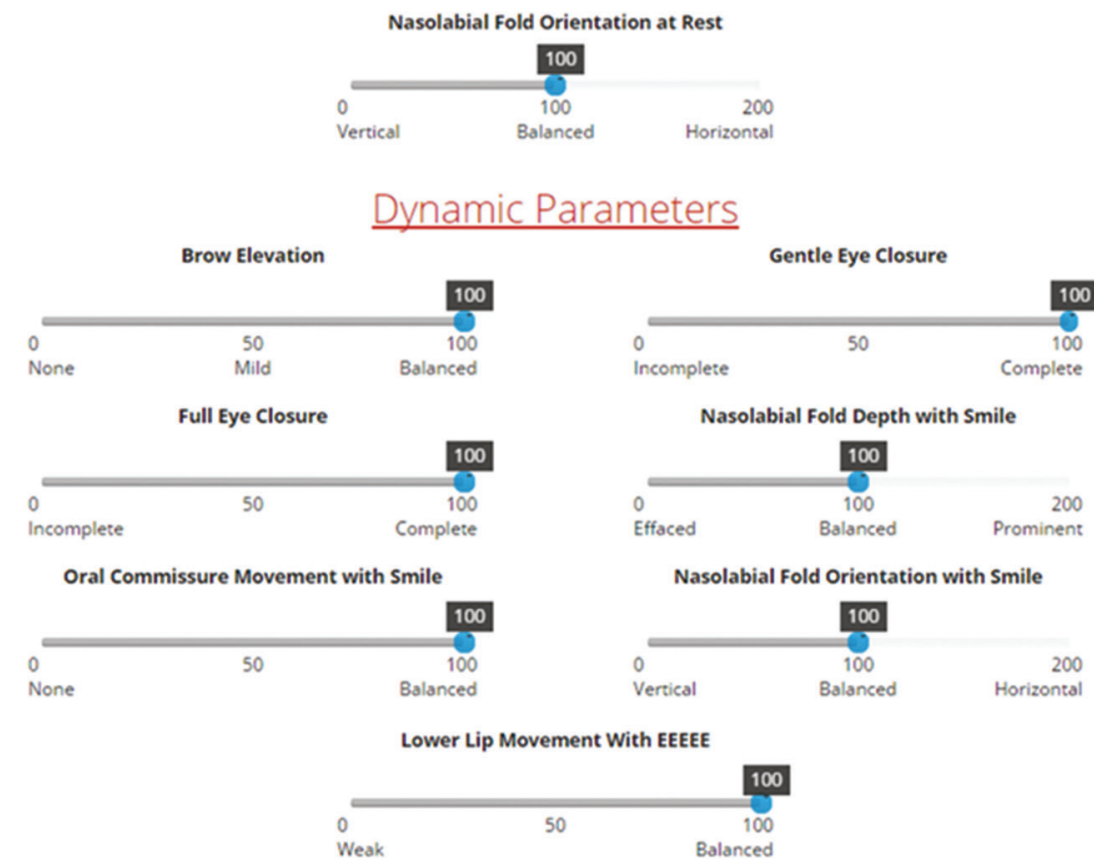

Synkinesis Parameters
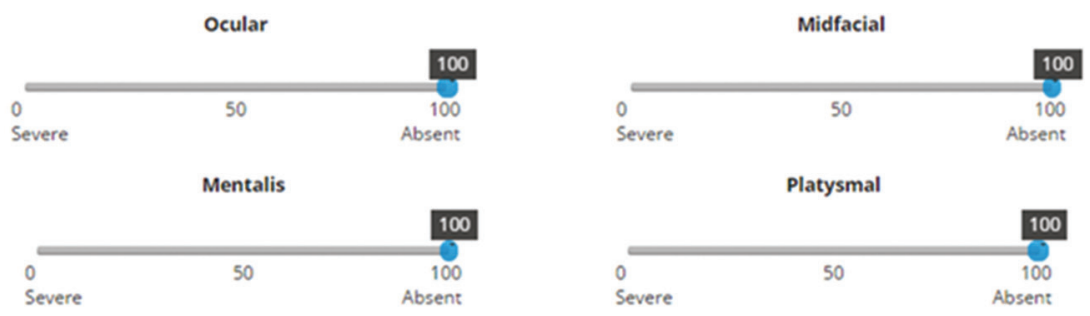

\section{View Results}

Fig. 5 The Facial Clinimetric Evaluation eFACE digital instrument that was designed to measure quality of life (QOL) impact of facial nerve disorders with three parameters: static, dynamic, and synkinesis, and can be accessed at http://links.Iww.com/PRS/B353. ${ }^{16}$ 
- Ocular-oral:

- Voluntary eyelid closure causes involuntary oral movement.

- Voluntary brow lift causes involuntary mouth angle lifting.

- Voluntary eye closure causes deepening of the nasolabial fold.

- Ocular-glabellar

- Platysmal (extremely common):

- Involuntary lower face and cervical contraction ensue with facial expressions.

- May result in downturn of the affected oral commissure (-Fig. 6).

- Mentalis synkinesis:

- Chin puckering with facial movement.

- Frequently evident as a mental dimple (-Fig. 6).

Importantly, synkinesis should only occur on the hemifacial side of the original injury. ${ }^{6}$

\section{BoNTA for Treatment of Asymmetry and Synkinesis}

Individualized injection patterns are necessary for optimal treatment of unilateral synkinesis and contralateral hyperkinesis (Fig. 7). Importantly, synkinesis treatment is aimed at accurate coordination of viable, synkinetic muscles rather than stimulating flaccid muscles, and insightful placement may improve animation patterns years after initial onset. ${ }^{6}$

BoNTA treatment also provides a "window of opportunity" for learning isolated, coordinated movement patterns (NMR) without restrictive synkinetic cocontraction. ${ }^{2}$ An

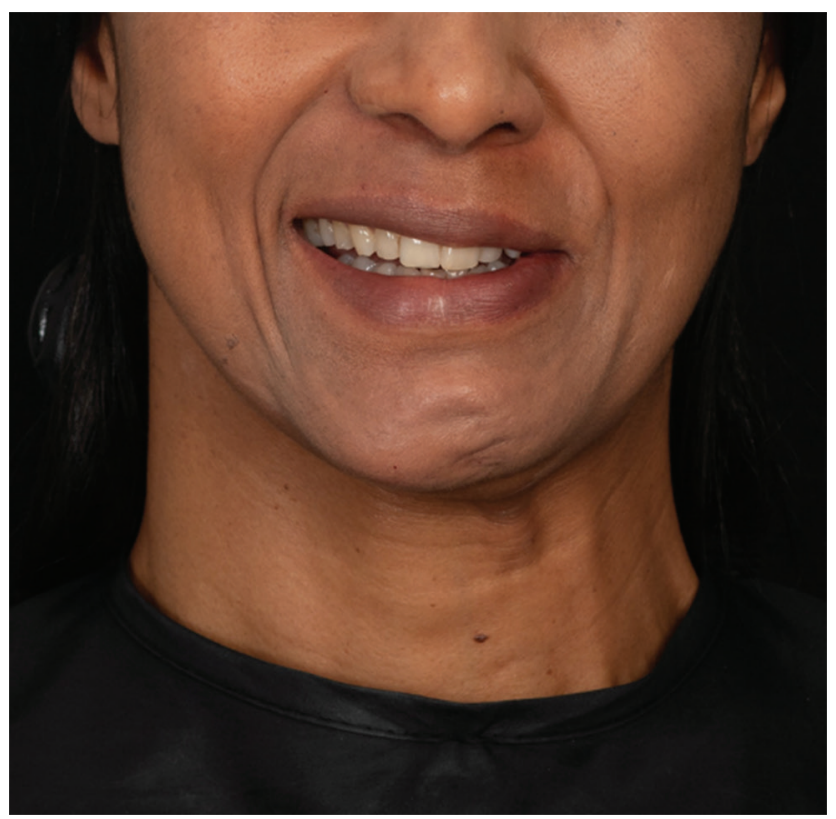

Fig. 6 Patient with left platysmal synkinesis, mentalis puckering, and downturned left oral commissure before treatment. initial 6 months of NMR is suggested before BoNTA to consolidate synkinesis inhibition techniques. ${ }^{6}$ The presence of BoNTA prior to retraining does not afford the same learning. ${ }^{6}$ Ipsilateral facial strength is frequently increased after contralateral botulinum toxin injection due to the 'strength redistribution phenomenon. ${ }^{18}$ This may be ascribed to either reduced dominance of hyperdynamic side or to central cortical reorganization after BoNTA. ${ }^{21}$ Accurately placed BoNTA decreases compensatory contralateral hyperkinesis and mediates more symmetrical function of the cheek, periocular, and perioral regions.

Treating contralateral, nonparalyzed muscles in conjunction with ipsilateral paralyzed muscles may result in long-lasting improvement of oral-ocular synkinesis; isolated ipsilateral injection generally causes only transient improvement. ${ }^{18}$ Fine, isolate muscle control on the paralyzed side gradually improves with diminishing strength on the hyperdynamic side. ${ }^{18}$ The treatment of orbicularis oculi and platysma may relieve synkinesis and spasm, while selective contralateral brow and depressor angularis oris injection may enhance facial symmetry and cosmesis. ${ }^{22}$ Improved synkinesis consistently leads to functional improvement with improved eye closure and smile ${ }^{19}$ ( - Tables 4 and $\mathbf{5}$ ).

Table 4 Grading system for synkinesis ${ }^{15}$

\begin{tabular}{|l|l|l|}
\hline Grade & Designation & Description \\
\hline 0 & None & No synkinesis or mass movement \\
\hline I & Mild & Minor synkinesis \\
\hline II & Moderate & $\begin{array}{l}\text { Evident but not disfiguring } \\
\text { synkinesis }\end{array}$ \\
\hline III & Severe & $\begin{array}{l}\text { Disfiguring synkinesis; significant } \\
\text { mass movement of numerous } \\
\text { muscles }\end{array}$ \\
\hline
\end{tabular}

Table 5 Synkinesis assessment questionnaire (SAQ) ${ }^{23}$

\begin{tabular}{|l|l|l|}
\hline & Question & Score \\
\hline 1 & When I smile, my eye closes & \\
\hline 2 & When I speak, my eye closes & \\
\hline 3 & When I whistle or pucker my lips, my eye closes & \\
\hline 4 & When I smile, my neck tightens & \\
\hline 5 & When I close my eyes, my face gets tight & \\
\hline 6 & $\begin{array}{l}\text { When I close my eyes, the corner of my mouth } \\
\text { moves }\end{array}$ & \\
\hline 7 & When I close my eyes, my neck tightens & \\
\hline 8 & When I eat, my eye waters & \\
\hline 9 & $\begin{array}{l}\text { When I move my face, my chin develops a dimpled } \\
\text { area }\end{array}$ & \\
\hline & $\begin{array}{l}\text { Total Synkinesis Score: Sum of Scores } \\
1 \text { to 9/45 X 100 }\end{array}$ \\
\hline
\end{tabular}

Questions answered on a scale from 1 to 5 , according to the following scale:

1 = seldom, or not at all.

2 = occasionally, or very mildly.

3 = sometimes, or mildly.

$4=$ most of the time, or moderately.

$5=$ all the time, or severely. 


\section{Neuromuscular Retraining (NMR)}

Facial NMR comprises a specialized subset of physical and occupational therapy aimed at improving motor learning and functional outcomes. ${ }^{17}$ The technique is based on the fact that the facial nerve innervates facial muscles devoid of muscles spindles, through emotional and volitional neural inputs, by utilizing sensory inputs to enhance neural adaptation. ${ }^{2}$ Minimal, precisely coordinated movement patterns are used in conjunction with surface proprioception, electromyography, and mirror feedback. ${ }^{24}$ Because synkinesis destroys normal facial muscle balance, apparent absence of movement may be due to abnormal restraining hyperactivity of opposing muscles. Knowledge of static anatomy is thus insufficient, as it is vital to grasp the dynamic action of individual muscles, both in isolation and in conjunction with others. ${ }^{6}$ Acquiring new motor behaviors via NMR stimulates neural plasticity and modifies the organizational capacity of the central nervous system. NMR is of great clinical value when combined with BoNTA. ${ }^{6}$

\section{Practical Guidelines}

In the author's practice, stable FNP is initially treated with conservative doses of BoNTA, according to a standardized injection scheme ( $\boldsymbol{\text { Fig. }}$ 7) to minimize side-effects. Patients are reviewed at 2 weeks for possible touch-up treatment, allowing opportunity for adjustment to altered muscle balance. During the initial visit, patients are encouraged to

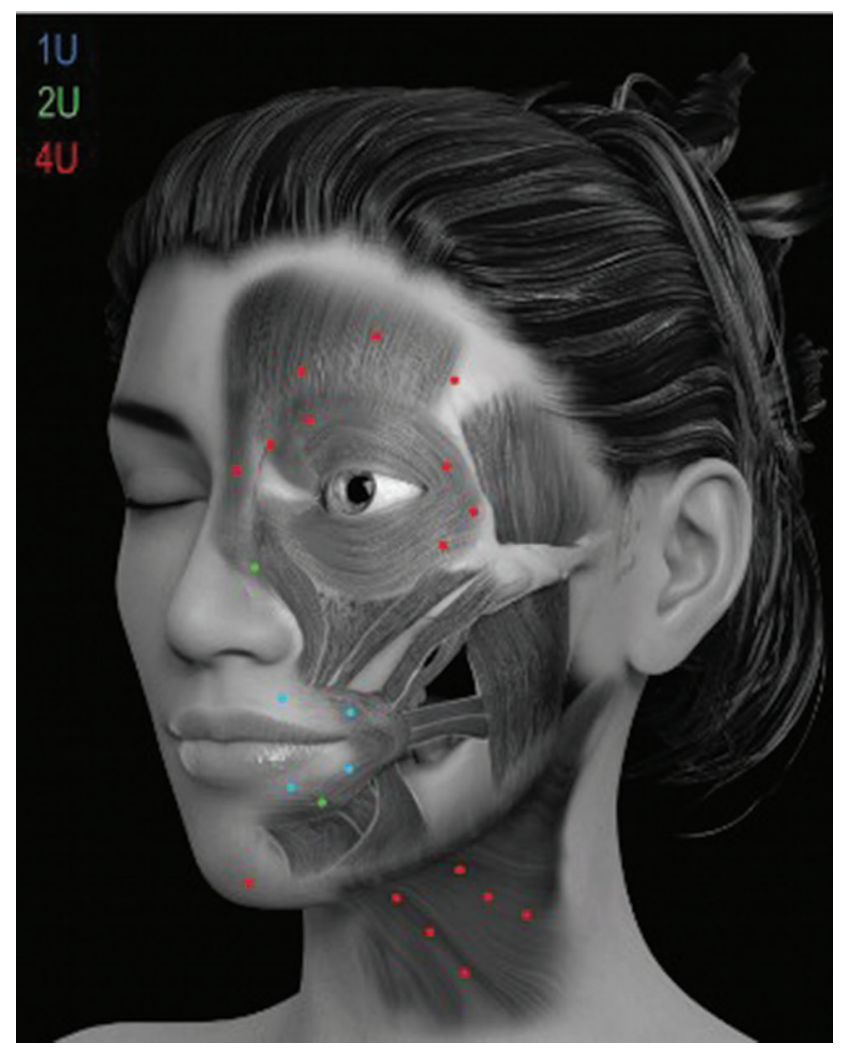

Fig. 7 Botulinum toxin (BoNTA) injection sites on the nonparalyzed side in relation to the facial muscles involved in acute facial palsy. Indicated doses are for onabotulinum toxin. ${ }^{5}$ chew on the weakened side, warned about potential oral incompetence and lip biting, and instructed in basic facial retraining exercises. A follow-up call at 48 hours reiterates instructions and offers encouragement, while a fortnightly appointment is scheduled for clinical follow-up, photographic documentation, and possible top-up treatment. Direct placement of BoNTA in weakened or synkinetic zygomaticus major and minor muscles are best avoided due to the debilitating effect of reduced smile function in a weakened face. ${ }^{4}$

However, due to the interdigitation of the orbicularis oculi and zygomaticus muscles, it may be possible to effectively treat ocular synkinesis by targeted low-dose BoNTA (2 U onabotulinum toxin/point) into the weakened, synkinetic orbicularis oculi.

Further treatment with hyaluronic acid (HA) fillers, according to the principles of myomodulation, is instituted one month after BoNTA in patients with suitable selection criteria for the use of HA fillers. ${ }^{25}$ In the author's practice, fillers are performed in patients with stable FNP at least 1 year after the acute event. Zygomaticus function and balance may be strengthened by insightful placement of HA fillers, with the functionality of the orbicularis oculi muscle enhanced by HA placement in the underlying medial and lateral suborbicularis oculi fat pad (SOOF). This causes an indirect, beneficial effect on the LPS. ${ }^{26,27}$

When treating patients with either BoNTA or HA fillers, it is imperative that injectors have a deep understanding of agonist and antagonist muscle groups, as levators and depressors work in opposition for normal, balanced facial expression (-Fig. 8). Understanding the position of the individual anatomical muscle layers is of paramount importance. While the optimal frequency of BoNTA treatment varies according to individualized needs, clinical practice verifies earlier studies

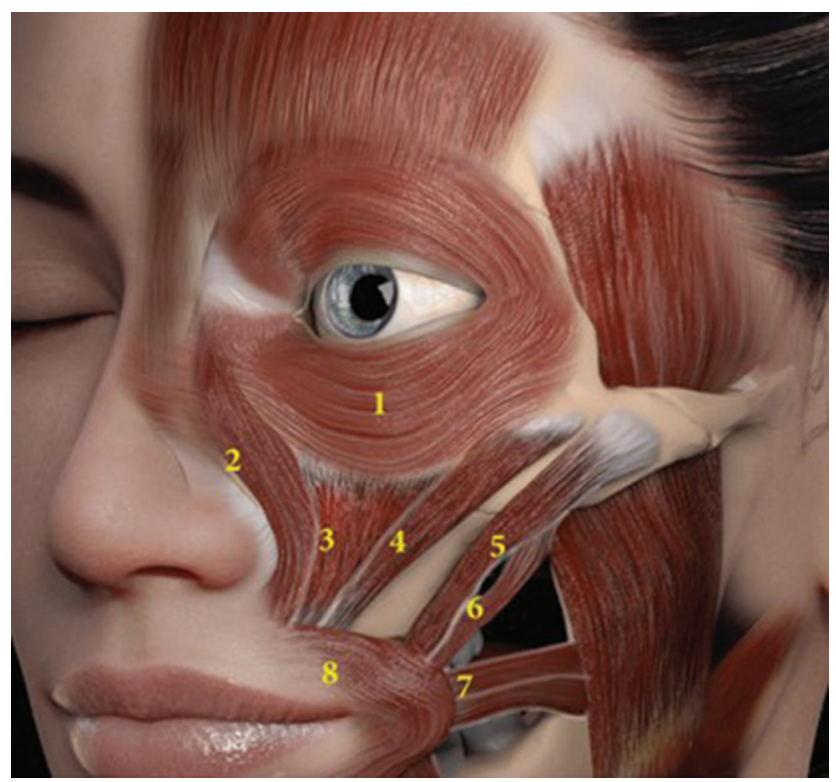

Fig. 8 Perioral muscles of animation. (1) Orbicularis oculi; (2) levator labii superioris alaeque nasi (LLSAN); (3) Levator labii superioris; (4) Zygomaticus minor; (5) Zygomaticus major; (6) Levator anguli oris; (7) Risorius; (8) Orbicularis oris (OO). ${ }^{5}$ 
in showing a 6-12 month duration of effect. ${ }^{26}$ When BoNTA is combined with HA fillers, improved baseline function and appearance may last beyond 1 year. Future randomized clinical trials are indicated in this regard.

Specific contraindications for the use of BoNTA in FNP include active infections, keratitis, symptomatic dry eye syndrome, myopathic or severe involutional ptosis, and myasthenia gravis. ( - Table 6 and - Fig. 9)

Table 6 Practical points for use BoNTA in FNP

- Document meticulously with assessment scales, photography, and videos both at rest and in animation.

- Assess for underlying residual facial nerve function on the palsy side, e.g., platysma and zygomaticus major.

- Treat the hyperdynamic side of the face with toxins to counter the Hering reflex; proceed conservatively in the perioral area to minimize functional discomfort.

- Follow-up at 2 weeks for possible top-up with toxins.

- Caution that phonation and chewing may initially be affected and warning against inadvertent lip biting and drooling.

- Encourage chewing on the weaker side in an attempt to recruit muscle strength.

- Myomodulation with fillers may be attempted in adequately selected patients at 1-month BoNTA treatment; fillers may be used to rebalance the face in cases of facial palsy, thus contributing significantly to QOL.

Abbreviations: BoNTA, botulinum toxin; FNP, facial nerve palsy; QOL, quality of life.

\section{Side Effects}

Adverse events occur more frequently with higher BoNTA doses and include oral incompetence, diplopia, ptosis, malaise, exposure keratopathy, and worsened cosmesis. ${ }^{28}$

Patients should be cautioned regarding altered phonation, inadvertent lip biting, and impaired chewing ability. They should be actively encouraged to chew on the weaker side and reminded that adverse events are short-term and reversible, while the long-term effects on contralateral muscle strength often outlast the duration of toxin action.

Previous studies cite a 50\% incidence of mild difficulty in drinking (without fluid loss), chewing (without food loss), phonation, and smiling at the 14-day follow-up visit. Thereafter, patients generally learn to coordinate the muscles, with a dramatic decrease in the rate of adverse events. ${ }^{26}$

\section{Future Trends}

Future trends for the management of FNP include the validated use of newly developed toxins, alternative methods for modifying muscle behavior, and improved imaging techniques. Topical daxibotulinumA gel (RT001) seems to hold possibilities for addressing superficial synkinetic muscles such as the orbicularis oris (OO). Injectable daxibotulinumA (RT002) promises more targeted delivery, less spread and a mean duration of 7 months, with positive practical implications. ${ }^{6}$

NMR is a valuable adjunct to BoNTA in treating synkinesis. Multidisciplinary treatment is most effective, with further studies necessary for standardizing and optimizing integrated

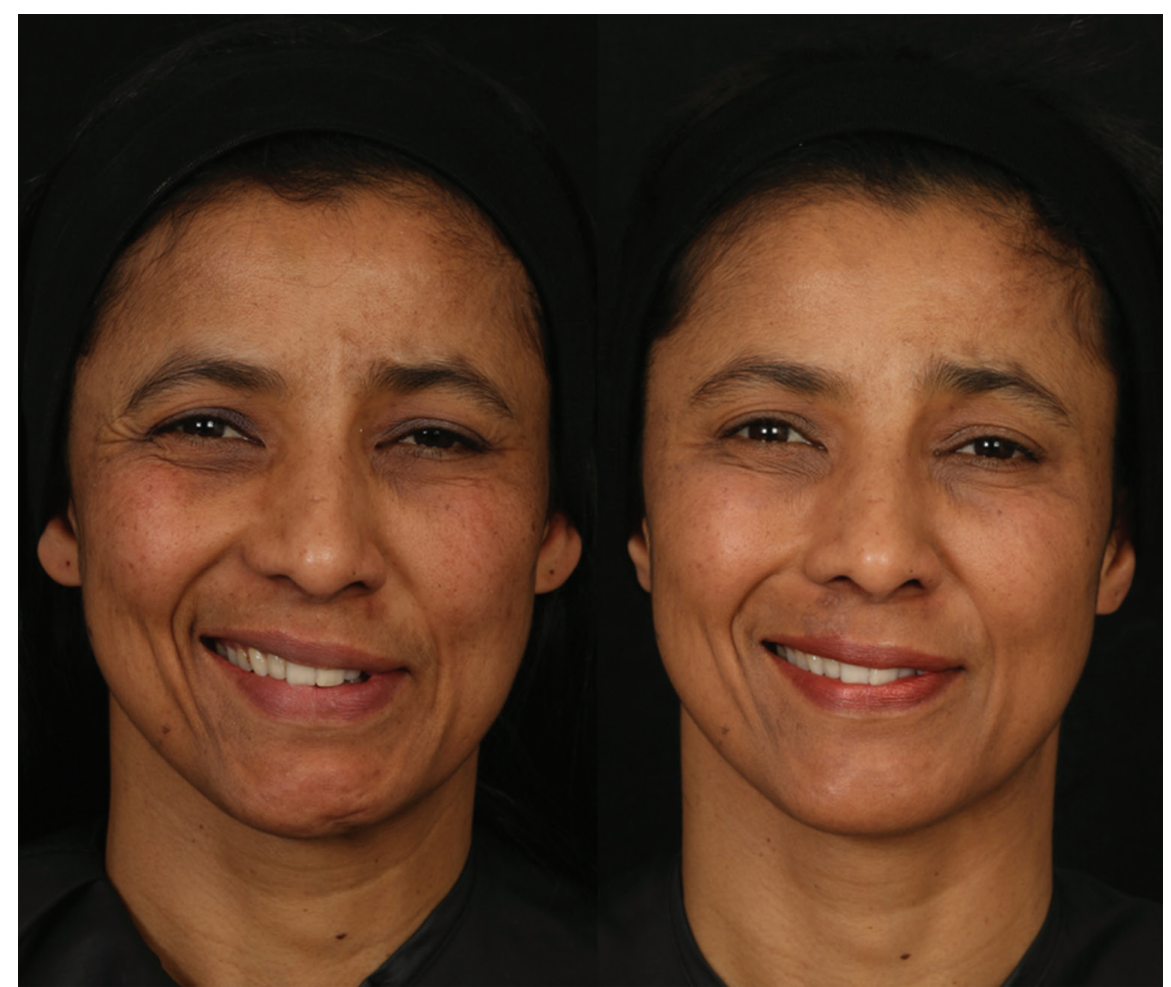

Fig. 9 Spontaneous animation before and two weeks after BoNTA to the mentalis, depressor labii inferioris (DLI), Orbicularis oris and Orbicularis oculi. Note the improvement in eye aperture on smiling; ocular synkinesis also improved dramatically. 
regimes. ${ }^{24}$ Filler techniques utilizing HA for modulating, facilitating, and rebalancing muscle movement according to the principles of myomodulation offer exciting new possibilities for managing FNP. 26,27

Injectable fillers are unique in that they can simultaneously support weak muscles, locally block overactive muscles, and enhance toxin efficacy through a fine-tuned balance between antagonist and synergist groups. ${ }^{26,27}$ Future value may be added through pre- and postfiller QOL assessments and by formally quantifying muscle action with simultaneous electromyography and videography.

Overall assessment of static, dynamic, and synkinetic parameters is currently being finessed utilizing electronic, zonally based, clinician-graded facial function scales. Threedimensional (3D) superimposition is evolving for assessment of facial reanimation procedures. ${ }^{20,29,30}$

Continuing research, with further evolution of multidisciplinary teams, will hopefully facilitate the development of objective dynamics, statistics, clinical guidelines, and procedural outcomes. ${ }^{2}$

\section{Conclusion}

The treatment goals of BoNTA in FNP include the reduction of synkinesis and establishment of a new dynamic balance between the paralyzed and nonparalyzed sides. Simple, systematic treatment schemes facilitate a stepwise approach, optimal dosing, and minimized side effects. BoNTA injections in acute FNP can lead to improvement for 6 months after a single treatment. As costs may impede adequate therapy in a patient cohort suitable for rehabilitation and return to socioeconomic functionality, establishment of NGOs could greatly alleviate treatment needs. It is hoped that a growing number of injecting physicians may develop interest, augment experience, and become involved in the multidisciplinary care of a patient cohort with great potential for improving QOL. One may echo Hippocrates in that, "We cure sometimes, treat often and comfort always."

\section{Conflicts of Interest}

None declared.

\section{Acknowledgments}

The author would like to express thanks to Elizabeth Rademan (BSc Honors Physiology, MA Journalism), Stellenbosch University, South Africa, for providing medical writing and editorial support for the manuscript.

\section{References}

1 Srivastava D, Singh H, Mishra S, Sharma P, Kapoor P, Chandra L. Facial asymmetry revisited: Part I- diagnosis and treatment planning. J Oral Biol Craniofac Res 2018;8(1):7-14

2 Cooper L, Lui M, Nduka C. Botulinum toxin treatment for facial palsy: a systematic review. J Plast Reconstr Aesthet Surg 2017;70(6):833-841

3 Kim S, Lee HY, Kim NK, Yook TH, Seo ES, Kim JU. The association between paralytic side and health-related quality of life in facial palsy: a cross-sectional study of the Korea National
Health and Nutrition Examination Survey (2008-2012) Health Qual Life Outcomes 2018;16(1):213

4 Eviston TJ, Croxson GR, Kennedy PGE, Hadlock T, Krishnan AV. Bell's palsy: aetiology, clinical features and multidisciplinary care. J Neurol Neurosurg Psychiatry 2015;86(12):1356-1361

5 Pirayesh A, Bertossi D, Heydenrych I, Aesthetic Facial Anatomy Essentials for Injections Boca Raton, Florida: CRC Press; 2020

6 Mehdizadeh OB, Diels J, White WM. Botulinum toxin in the treatment of facial paralysis. Facial Plast Surg Clin North Am 2016;24(1):11-20

7 Tavares-Brito J, van Veen MM, Dusseldorp JR, Bahmad F, Jr, Hadlock TA. Facial palsy-specific quality of life in 920 patients: correlation with clinician-graded severity and predicting factors. Laryngoscope 2019;129(1):100-104

8 Kim J. Contralateral botulinum toxin injection to improve facial asymmetry after acute facial paralysis. Otol Neurotol 2013;34(2):319-324

9 Zhang W, Xu L, Luo T, Wu F, Zhao B, Li X. The etiology of Bell's palsy: a review. J Neurol 2019;(e-pub ahead of print). doi: 10.1007/s00415-019-09282-4

10 Terzis JK, Bruno W. Outcomes with eye reanimation microsurgery. Facial Plast Surg 2002;18(2):101-112

11 Terzis JK, Noah ME. Analysis of 100 cases of free-muscle transplantation for facial paralysis. Plast Reconstr Surg 1997;99(7):1905-1921

12 Sinha KR, Rootman DB, Azizzadeh B, Goldberg RA. Association of eyelid position and facial nerve palsy with unresolved weakness. JAMA Facial Plast Surg 2016;18(5):379-384

13 Tan P, Kwong TQ, Malhotra R. Non-aesthetic indications for periocular hyaluronic acid filler treatment: a review. $\mathrm{Br} \mathrm{J}$ Ophthalmol 2018;102(6):725-735

14 House JW, Brackmann DE. Facial nerve grading system. Otolaryngol Head Neck Surg 1985;93(2):146-147

15 Ross BG, Fradet G, Nedzelski JM. Development of a sensitive clinical facial grading system. Otolaryngol Head Neck Surg 1996;114(3):380-386

16 Banks CA, Bhama PK, Park J, Hadlock CR, Hadlock TA. Cliniciangraded electronic facial paralysis assessment: The eFACE. Plast Reconstr Surg 2015;136(2):223e-230e

17 Robinson MW, Baiungo J. Facial rehabilitation: evaluation and treatment strategies for the patient with facial palsy. Otolaryngol Clin North Am 2018;51(6):1151-1167

18 Choi KH, Rho SH, Lee JM, Jeon JH, Park SY, Kim J. Botulinum toxin injection of both sides of the face to treat post-paralytic facial synkinesis. J Plast Reconstr Aesthet Surg 2013;66(8):1058-1063

19 Terzis JK, Karypidis D. Therapeutic strategies in post-facial paralysis synkinesis in adult patients. Plast Reconstr Surg 2012;129(6):925e-939e

20 White DM. The "Gate Keeper": a case study in the selection of news. Journal Bull 1950;27(4):383-390

21 Choi YJ, Lee WJ, Lee HJ, Lee KW, Kim HJ, Hu KS. Effective botulinum toxin injection guide for treatment of temporal headache. Toxins (Basel) 2016;8(9):1-10

22 Cheong YW, Lo LJ. Facial asymmetry: etiology, evaluation, and management. Chang Gung Med J 2011;34(4):341-351

23 Mehta RP, WernickRobinson M, Hadlock TA. Validation of the synkinesis assessment questionnaire. Laryngoscope 2007;117(5):923-926

24 van Landingham SW, Diels J, Lucarelli MJ. Physical therapy for facial nerve palsy: applications for the physician. Curr Opin Ophthalmol 2018;29(5):469-475

25 Heydenrych I, Kapoor KM, De Boulle K, et al. A 10-point plan for avoiding hyaluronic acid dermal filler-related complications during facial aesthetic procedures and algorithms for management. Clin Cosmet Investig Dermatol 2018;11(11):603-611 
26 de Maio M. Myomodulation with Injectable Fillers: An Innovative Approach to Addressing Facial Muscle Movement. Aesthetic Plast Surg 2018;42(3):798-814

27 De Maio M, Heydenrych I, Myomodulation. In: Pirayesh A, Bertossi D, Heydenrych I, eds Aesthetic Facial Anatomy Essentials for Injections. Boca Raton, Florida: CRC Press; 2020

28 Kahn JB, Gliklich RE, Boyev KP, Stewart MG, Metson RB, McKenna MJ. Validation of a patient-graded instrument for facial nerve paralysis: the FaCE scale. Laryngoscope 2001;111(3):387-398
29 Wollina U, Goldman A. Botulinumtoxin-A und dermale Gewebefiller in der fazialen rehabilitation. Wien Med Wochenschr 2017;167(3-4):92-95

30 Sforza C, Ulaj E, Gibelli DM, et al. Three-dimensional superimposition for patients with facial palsy: an innovative method for assessing the success of facial reanimation procedures. $\mathrm{Br} \mathrm{J}$ Oral Maxillofac Surg 2018;56(1):3-7 